\title{
Hybrid Network of Neuro-Fuzzy based Decision Tool for Stock Market Analysis
}

\author{
J. Kumaran Kumar \\ Research scholar in SCSVMV \\ university \\ Assistant professor \\ Department of Computer \\ Science and Engineering, \\ Pondicherry Engineering \\ College,Puducherry, India
}

\author{
G. Ravi, PhD. \\ Associate professor \\ Department of Electrical and \\ Electronics Engineering \\ Pondicherry Engineering \\ College \\ Puducherry, India
}

\author{
T.Mugilan \\ PG student \\ Department of computer \\ science and engineering \\ pondicherry engineering \\ college puducherry.india
}

\begin{abstract}
Prediction of stock market return is an important issue in finance. Fuzzy and Artificial neural networks have been used in stock market prediction during the last decade. Studies were performed for the forecast of stock index values as well as daily direction of change in the index. This work compares fuzzy and arrangement of ANN model and makes these models to train with the past 5 years stock price datasets of various companies like (TCS, HCL) and the prediction of future stock price of company has been found. Membership functions (LOW, MEDIUM, HIGH) based fuzzy model will give recommendation for investor which says the current situation of the stock market. The Root Mean Square error (RMSE), Mean Absolute Performance (MAPE) metrics calculates the error rate value of each model. The proposed Hybrid Network model has expecting to given high performance.
\end{abstract}

\section{KEYWORDS}

Fuzzy, ANN, RMSE, MAPE, Index, Datasets

\section{INTRODUCTION}

The paper describes the use of soft computing as a decision making support for long-term venture on the financial markets. It is very difficult to predict the growth of financial markets $[1,2]$. Markets are active and there are many intricate factors and complicated relationships that influence indexes, currencies and merchandise which makes investing intricate and uncertain. The process in financial system has nonlinear factors. If the system is nonlinear and dynamic, it can engender randomly looking behavior but it can include the permanent trends and cycles. Investing on the financial markets is tricky because of globalized economies - there are different crisis, bubbles, rising debts and prices of merchandise, energy etc. These problems arbitrarily escalate and create extreme imbalance on the market. These imbalances created have great opportunity and threats for the investors. Psychology also plays an important mission on the financial markets - investors often do not recognize these opportunities because they are scared of the future growth. This research is facing very actual and yet at the same time classic problem of investing -when to buy and sell stocks while minimizing the exposure. Accepting the markets and being able to predict what will happen in the near future are the key skills that every unbeaten investor must keep in mind. This research uses a simple model with a few variables that simplify the complex market atmosphere to make consistent recommendations for the investors and so provides a valuable decision making support tool.
Stock market $[1,2]$ is an important support of each economy. Share is the control of the company divided into small parts and each part is called as Share or Stock. A stock exchange is a firm or mutual union which provides "trading" services for traders and stock brokers, to trade stocks and other securities. A trader who is looking to profit from the market has to have belief on the company where he is putting his money. Technical analysis uses price and other similar daily input data, and the elementary analyst's uses fundamental data which are normally published on an annual basis or quarterly. Technical and fundamental approaches are used in parallel to educated guess short-term and long-term return and risk.

Fuzzy Logic framework is developed to carry out the required analysis for arriving at the governance rating of the compact. This study will help the customer for decision making in share market [3.5]. A person carrying a share of a company holds that part of ownership in that company .Share is nothing but the Ownership of the company divided into small parts and each part is called as Share otherwise called Stock. Share market is the place where buying and selling of shares takes place. The buying and selling of shares takes place from anywhere, with the assist of internet connected computer. One should need the demat account, computer and internet connection and that is required to start the share trade or investing. When you place the buy order in India, the communication is transferred to the exchange [either NSE \{National Stock Exchange\} or BSE \{Bombay Stock Exchange \}] and the order stays in the queue of exchange's other orders and gets executed if the price of that share comes to that charge. Once you get the statement/confirmation of this transaction the shares purchased will be sent to his/her demat account. In electronic design the shares will be stored. The Sensex is an "index". An directory is an indicator that gives us a general idea about whether most of the stocks have gone up or down. On considering India the Sensex is an pointer of all the foremost companies of the BSE and the Nifty is an indicator of NSE. If the Sensex goes up, it means that the prices of the stocks of most of the major companies on the BSE have gone up. If the Sensex goes down, it tells us that the stock price of most of the major stocks on the BSE has gone-down. Thus the Sensex represent the top stocks of the BSE and the Nifty represents the top stocks of the NSE. Besides Sensex and the Nifty there are many other indexes and many different types of investors hold the shares of a company.

There are two major approaches to the analysis of stock market price prediction: Fundamental analyses and technical analyses [9, 10]. Fundamental analysis is the move toward of studying the overall economy, industry, financial conditions and management of companies to measure the intrinsic value of a particular 
security. This approach uses revenues, earnings, future development and returns on fairness, return margins, and other data to establish a company's underlying value and the potential for future growth of its security. Technical analysis, on the other hand, does not challenge to measure a security's intrinsic value. This approach evaluates securities by analyzing statistics generated by market activity, such as past prices and quantity. The pioneering technical analysis technique is attributed to $\mathrm{C}$. The efficient market hypothesis (EMH) is generally interpreted to imply that the technical approach to forecasting stock price is invalid, but recent reporting presented from a behavioral finance perspective and statistical inference from computational algorithms further exemplify the evidence on the inevitability of financial market using technical analysis. Thus technical analysis $[6,7,8]$ has recently enjoyed a renaissance and most major brokerage firms publish technical commentary on the market and individual securities.

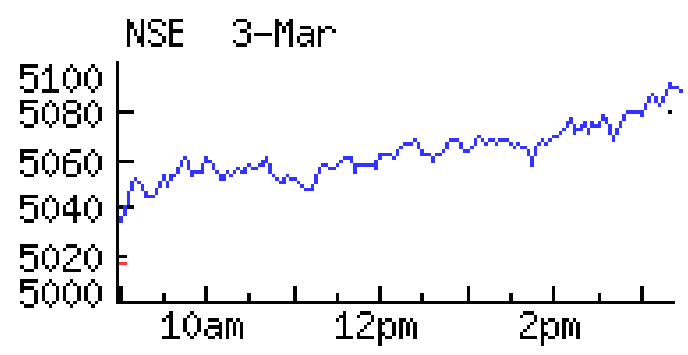

Fig. 1 NSE graph

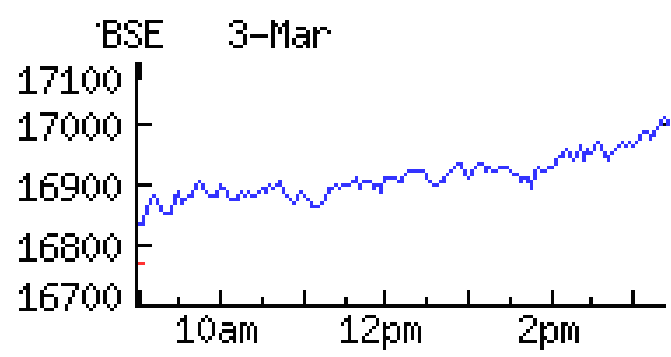

Fig. 2 BSE graph

The main approach in financial forecasting is to recognize trend at an early stage in order to keep up an investment strategy until evidence indicates that the trend has reversed. Predictability of protection from past real-world data using two of the simplest and most popular trading rules, namely moving average and trading range break-out rules. Other techniques used include regression methods and the ARIMA models, but these models fail to give satisfactory forecast for some series because of their linear structures and some other inherent limitations. Although there are also ARCH/GARCH models to deal with the non constant variance, these models also fail to give satisfactory forecast for some. Increasingly applications of artificial intelligence (AI) techniques, mainly artificial neural networks, have been applied to technical financial forecasting as they have the ability to learn complex nonlinear mapping and selfadaptation for different statistical distributions .An investigation on the nonlinear predictability of security returns from past realworld returns using single layer feed-forward neural network and moving average rules was presented in on the Stock Market. The results showed that evidence of nonlinear predictability in stock market returns can be found by using the past buy and sell signals of the moving average rules. Application of AI techniques in financial forecasting is not restricted only to the technical analysis approach, but has also been applied to the fundamental approach. For example, in the work of, a genetic algorithm based fuzzy neural network is trained with additional political, financial, economic factors etc. to formulate trading decisions. A number of research investigations have been published on the application of AI techniques in the technical analysis approach of forecasting stock price, but only a few offered quantitative results on trading performance using real world stock market facts. The performed analysis of predictability based on a history of closing price of a number of elevated unpredictability stocks and consumer stocks using time delay, persistent and probabilistic neural networks.

\section{RELATED WORKS}

A hybrid network is planned with a trigonometric Functional Link Artificial Neural Network (FLANN) and Fuzzy Logic System which is named as Functional Link Neural Fuzzy (FLNF) Model $[3,4,8,9]$ both of which is used to predict the stock market indices. Proposed work is designed with a functional link neural network to the consequent part of the fuzzy rules. The subsequent part of FLNF model is a non-linear mixture of input variables. Two stock market indices that is, the dataset of Bombay Stock Exchange and Standard's and Poor's (S\&P500) are collected for experimentation and samples for 4000 trading days from $1 \mathrm{st}$ March 1993 to 23rd July 2009 are collected earlier and 3228 trading days from $1^{\text {st }}$ March 1993 to 09th June 2006 for the later. This model is used to calculate the stock market indices for one day, one week and one month in advance. This also gives the comparative analysis between the proposed hybrid model and that of FLANN, MAPE and RMSE are used to find out the performance of both the models and it shows the superiority of the hybrid model. Simulation results have demonstrated better performance of FLNF model compared to FLANN. As back propagation learning algorithm is used to optimize the model parameters of both models, the result may reach the local minima problem [10, 11]. To overcome this limitation some global optimization technique like Particle Swarm Optimization may be used to optimize the parameters. Secondly more number of indicators can also be included to improve the performance of the FLNF model.

Analysis of Input Datasets:

\section{Sales Turnover}

2. Earning Per Share (EPS)

3. Dividend

4. Book Value

5. Price Earning Ratio (P/E)

\subsection{Sales Turnover}

The total amount sold within a specific time period, usually a year. Sales turnover is often expressed in monetary terms but can also be expressed in terms of the total amount of stock or products sold.

Sales Turnover=Total Sales turnover/Total sales turnover average.

\subsection{Earnings Per Share:}

EPS plays major role in investment decision. EPS is calculated by taking the net earnings of the company and dividing it by the outstanding shares. 
EPS $=$ Net Earnings $/$ Outstanding

Earnings per Share=Total EPS/ Total EPS average.

\subsection{Dividend (\%)}

A distribution of a portion of a company's earnings, decided by the board of directors, to a class of its shareholders. The dividend is most often quoted in terms of the dollar amount each share receives (dividends per share). It can also be quoted in terms of a percent of the current market price, referred to as dividend yield.

Dividend=Total Dividend/ Total Dividend average.

\subsection{Book Value}

Book value is the total value of the company's assets that shareholders would theoretically receive if a company were liquidated (closed). By being compared to the company's market value, the book value can indicate whether a stock is under priced or overpriced. So in other words if the share price is trading below its book value then it is considered as under priced and good for value invest.

Book value $=$ Total Book value $/$ Total Book value average.

\section{$2.5 \quad(P / E)$ Price Earning Ratio}

$\mathrm{PE}$ ratio is again one of the most important ratio on which most of the traders and investors keep observe. The PE ratio tells you whether the stock's price is high or low compared to its familiar. The high $\mathrm{P} / \mathrm{E}$ suggests that investors are expecting higher earnings growth in the future compared to companies with a lower P/E. This generally happen in bull market and share price keeps on increasing. Basically in bull market share prices keep increasing without giving more importance to its current valuation and once market realizes that it is dear then they start selling. In bear market the low PE stocks having high enlargement prospects are selected as best investment options. But, the P/E ratio doesn't tell us the whole story of the company. Generally the P/E ratios are compared of one company to other companies in the same sector/industry and not in other industry before selecting any particular share

$\mathrm{P} \backslash \mathrm{E}=$ Total average $/(\mathrm{ple})$.

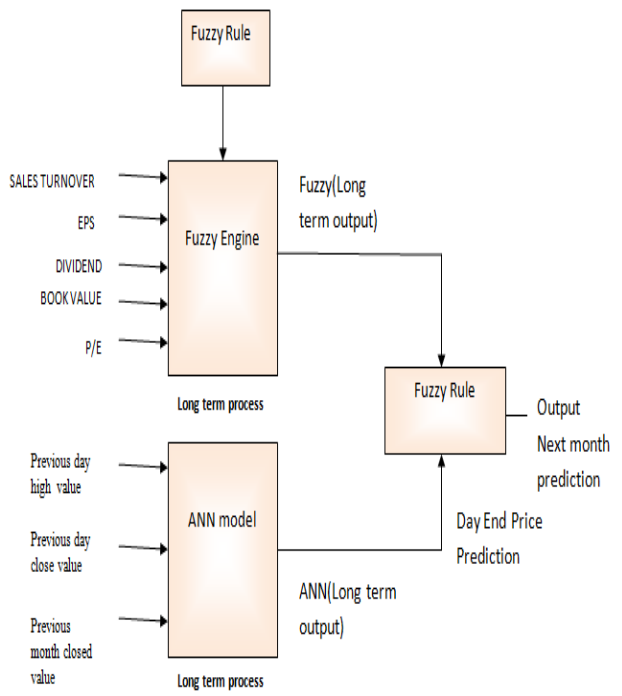

Fig. 3 Proposed Hybrid Network Model (Fuzzy\& ANN model)

\section{PROPOSED WORK}

Neuro-fuzzy refers to aggregation of artificial neural networks and fuzzy logic. Hybrid intelligent system as a effect of Neurofuzzy hybridization that synergizes these two techniques by combining the human-like analysis style of fuzzy systems with the learning and connectionist structure of neural networks. Neuro-fuzzy hybridization is widely termed as Fuzzy Neural Network (FNN) or Neuro-Fuzzy System (NFS). Neuro-fuzzy system incorporates the human-like reasoning style of fuzzy systems through the use of fuzzy sets and a linguistic model consisting of a set of IF-THEN fuzzy conventions. The main strength of neuro-fuzzy systems is that they are universal approximators with the ability to solicit interpretable IF-THEN rules.

Fuzzy model is developed with following five inputs sales turnover, EPS, Dividend, Book value and P/E in fuzzy engine. Fuzzy rule can be generated, based on membership function. It recommends to investor whether to buy a particular share or not. To predict the day end prediction following three inputs previous day high value, previous day close value and previous month closed value are given in ANN model. Finally fuzzy engine and ANN model is integrated with new Fuzzy rule to predict the next month close price prediction.

The proposed Neuro Fuzzy based decision tool move in the direction of learns the rules and membership functions from data. Neuro Fuzzy is an adaptive network that is a network of nodes and directional links. One of the Neuro Fuzzy reward is that it uses a hybrid learning procedure for estimation of the premise and resultant parameters. Using a fuzzy inference system in the framework of an adaptive neural network, it provides a tool which make to grade certainty more accurate since by using both neural network and fuzzy logic. The concert of the model with respect to the predictions made on the test data set would able the network to execute more accurate than preceding methods. The hybrid Neuro-Fuzzy decision Tool is shown in the Fig 3 and Sample Inputs are tabulated in Table 1.

\subsection{Generation of Rules for Fuzzy Model}

Consider value as,

Low $=1$

Medium=2

High $=3$

High $=[3,3,3,3,3]$ (Strong buy)

Medium $=[3,2,3,3,2]($ Based on situation (buy or sell))

Low $=[1,2,2,1,1]($ Strong sell $)$ LOW MEDIUM HIGH

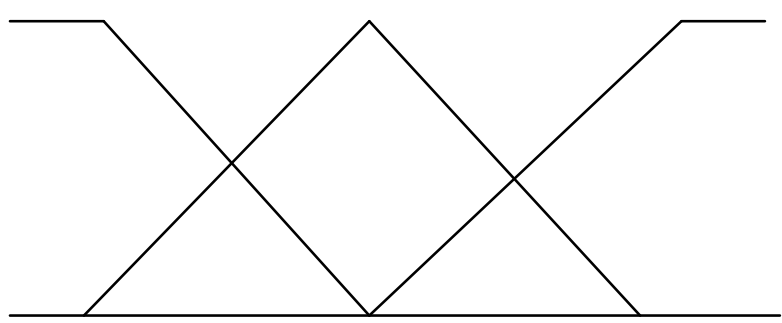

0

0.35

0.5

Fig 2 Sample Membership Function For Fuzzy Logic 
Inputs for Fuzzy Model

1. Sales Turnover $=[$ Low, Medium ,High $]$

2. Earnings per Share=[Low ,Medium ,High]

3. Dividend $(\%)=[$ Low ,Medium ,High $]$

4. Book value $=[$ Low, Medium, High $]$

5. PLE (Price earnings ratio) $=[$ Low ,Medium ,High $]$

\subsection{ANN Model}

Inputs for ANN Model

1. Previous Day High Value

2. Previous Day Close Value

3. Previous Month Closed Value

TABLE I. FUZZY RULES GENERATION

\begin{tabular}{|l|l|l|l|l|l|l|}
\hline $\begin{array}{l}\text { Compani } \\
\text { es }\end{array}$ & $\begin{array}{l}\text { Inp } \\
\text { ut1 }\end{array}$ & $\begin{array}{l}\text { Inp } \\
\text { ut 2 }\end{array}$ & $\begin{array}{l}\text { Inp } \\
\text { ut3 }\end{array}$ & $\begin{array}{l}\text { Inp } \\
\text { ut 4 }\end{array}$ & $\begin{array}{l}\text { Inp } \\
\text { ut 5 }\end{array}$ & Result \\
\hline TCS & High & High & High & High & Med & $\begin{array}{l}\text { Strong } \\
\text { Buy }\end{array}$ \\
\hline HCL & High & High & High & Med & High & $\begin{array}{l}\text { Strong } \\
\text { Buy }\end{array}$ \\
\hline DLF & High & High & High & Low & High & $\begin{array}{l}\text { Strong } \\
\text { Buy }\end{array}$ \\
\hline MRF & High & High & Med & Med & Low & $\begin{array}{l}\text { (Buy Or } \\
\text { Sell) }\end{array}$ \\
\hline WIPRO & High & Low & High & Med & High & $\begin{array}{l}\text { (Buy Or } \\
\text { Sell) }\end{array}$ \\
\hline SUZLON & Low & High & Low & Low & High & $\begin{array}{l}\text { Strong } \\
\text { Sell }\end{array}$ \\
\hline
\end{tabular}

\subsection{Performance Metric}

1. Less error rate

$$
R M S E=\sqrt{\frac{1}{N} \sum_{k=1}^{N}\left(t_{k}-o_{k}\right)^{2}}
$$

where $t_{k}$ is predicted value

$o_{k}$ is desired value

$N$ is the total number of test data.

2. More accuracy

$$
M A P E=\frac{1}{n} \sum_{t=1}^{n}\left|\frac{A_{t}-F_{t}}{A_{t}}\right| * 100
$$

Where $A_{t}$ is predicted value

$F_{t}$ is desired value.

\begin{tabular}{|c|c|c|c|c|c|c|}
\hline \multirow[t]{2}{*}{ COMPANYS } & \multicolumn{3}{|c|}{ MAPE } & \multicolumn{3}{|c|}{ RMSE } \\
\hline & FUZZY & ANN & HYBRID & FUZZY & ANN & $\begin{array}{l}\text { HYB } \\
\text { RID }\end{array}$ \\
\hline TCS & $82 \%$ & $90 \%$ & $98 \%$ & 0.63 & 0.13 & 0.02 \\
\hline HCL & $84 \%$ & $92 \%$ & $98.2 \%$ & 0.65 & 0.23 & 0.03 \\
\hline INFOSYS & $84.5 \%$ & $93 \%$ & $97.6 \%$ & 0.62 & 0.27 & 0.04 \\
\hline ACCENTURE & $86 \%$ & $94 \%$ & $99 \%$ & 0.79 & 0.30 & 0.02 \\
\hline ARICENT & $82 \%$ & $92 \%$ & $98 \%$ & 0.69 & 0.15 & 0.05 \\
\hline
\end{tabular}

TABEL II.COMPARISON ANALYSIS
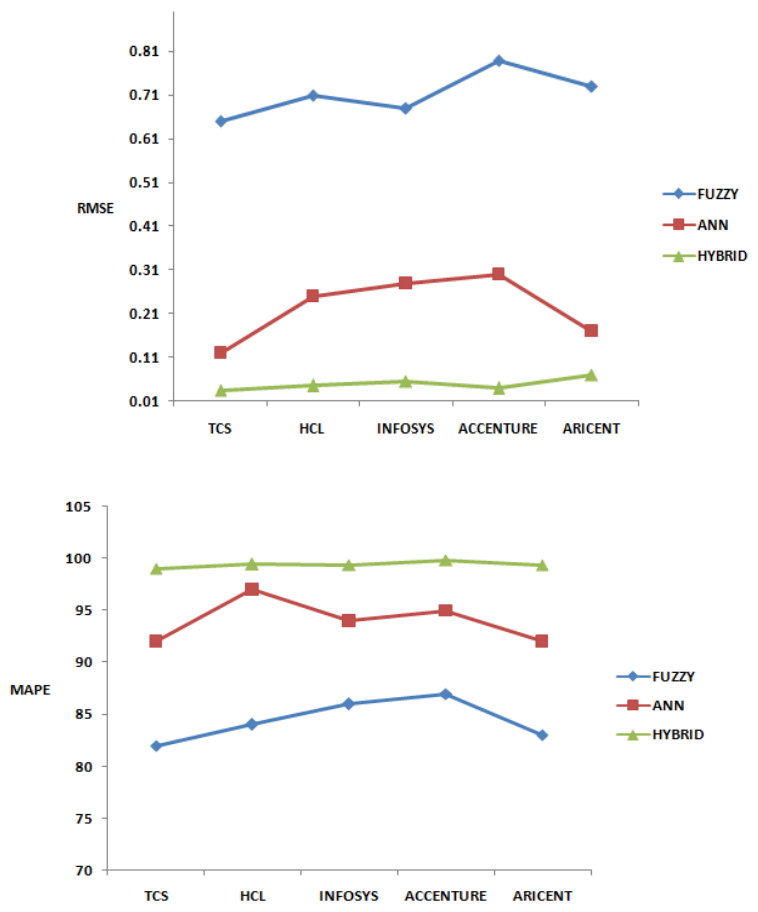

\section{CONCLUSION}

The two models for decision making support based on the fuzzy and hybrid neuro-fuzzy model have been develop and implemented with 200 days of historical data. To design model has been tested extensively with the historical data and it has proved to provide correct investment recommendations with high statistical probability. To conclude that hybrid neuro fuzzy model is more efficient and more accurate in term of error than fuzzy model for forecasting analysis. The design model is useful for investors who are looking for a path to manage risk when dealing with the long-term investment portfolio and better provide recommendations for the investor, limit risk and Easley identify opportunities. 


\section{REFERENCES}

[1] Chakravarty S, and Dash P.K., "Forecasting Stock Market Indices Using Hybrid Network" 78-1-4244-56123/09/\$26.00_c IEEE 2009.

[2] Chakravarty S, Mohapatra P and Dash P.K., " Stock Market Prediction using Dynamic Filter Weights Neural Network and Paticle Swarm"- FFMI, -VGSOM, IIT, Khargpur 2008.

[3] Chen Chen-Hung, “ A Functional-Link-Based Neurofuzzy Network for Nonlinear System Control"- IEEE Transaction on Fuzzy Systems, Vol 16 No 5, October 2008.

[4] Ivna Valenc,a and Teresa Ludermir, M^euser Valenc, a "Hybrid Systems to Select Variables for Time Series Forecasting Using MLP and Search Algorithms"- 978-0$7695-4210-2 / 10 \quad \$ 26.00 \quad$ (C) $2010 \quad$ IEEE DOI $10.1109 /$ SBRN.2010.

[5] Chen Yuehui, Yang Bo,Dong Jiwen, "Time Series Prediction using a local linear wavelet neural network". Neuro computing 69 (2006) 449-465, 2005.

[6] Chen Yuehui, Dong Xiaohui, Zhao Yaou: "Stock Index Modelling using EDA based Local Linear Wavelet Netowork". IEEE. 2005.
[7] Chodhury Rohit \& Garg Kumkum, "A Hybrid Machine Learning System for Stock Market Forecasting" Proceeding of World 2009.

[8] S. M. Chen and N. Y. Chung, "Forecasting enrollments using high-order fuzzy time series and genetic algorithm," Int. J. Intell. Syst., vol. 21, no. 5, pp. 485-501, May 2006.

[9] S. M. Chen and H. R. Hsiao, "A new method to estimate null values in relational database systems based on automatic clustering techniques," Inf. Sci., vol. 169, no. 1/2, pp. 47-69, Jan. 2005.

[10] T. Plummer and A. Ridley, "Forecasting Financial Markets: The Psychology of Successful Investing", London, U.K.: Kogan, 2003.

[11] K. Huarng and H. K. Yu, "Ratio-based lengths of intervals to improve fuzzy time series forecasting," IEEE Trans. Syst., Man, Cybern. B, Cybern., vol. 36, no. 2, pp. 328-340, Apr 2006. 